\title{
Model Reference Adaptive Controller for LTI Systems with Time-variant Delay
}

\author{
Ghedjati Keltoum \\ Department of Electrical Engineering \\ University Ferhat Abbas Setif 1 \\ Setif, Algeria \\ ghedjat_keltoum@yahoo.com
}

\begin{abstract}
In this paper, a new Direct Model Reference Adaptive Control Procedure (DMRAC) for Linear Time-Invariant (LTI) delay systems is presented with the use of the concept of the command generator tracker which expands the class of processes that can now be controlled with zero output error. The stability of the error between the system and the model is guaranteed by the Lyapunov theory. The new algorithm is applied to control a perturbed delay system. Matlab simulation examples are given to demonstrate the usefulness of the algorithm.
\end{abstract}

Keywords-adaptive control; asymptotic stability; time delay systems; dynamical uncertainties

\section{INTRODUCTION}

The stability of time delay systems has been studied with the Lyapunov-Krasovskii and the Lyapunov-Razumikhin approach. These two concepts have been used in order to avoid the classical Lyapunov method. Authors in [1-3] give an overview of the stability of time delay systems with some advanced results. The rightmost roots of the characteristic are investigated in [4]. Authors in [5] studied the control of an MIMO nonlinear time delay system. Stability analysis and stabilization for Takagi-Sugeno (T-S) fuzzy systems with time delay have been studied in $[6,7]$. In [8], a delay-dependent stabilization condition was proposed for the stability of a class T-S fuzzy time-delay system using homogeneous polynomials scheme and Polya's theorem with application on a truck-trailer model. Authors in [9] investigated the pre-specified performance for time-varying delays using model reduction, fuzzy logic, and LMI techniques. The PID controller has also been used in the stability of the time-delay systems [10]. The developed method guarantees gain and phase margins besides stability.

The introduction of adaptive control in uncertain time delay systems has been studied thoroughly. In [11], the author used the back stepping transformation where regulation was achieved despite the presence of partial measurements and disturbance. The adaptive identification of the parameters and the time delay of the time delay system were addressed in [12]. This identification is achieved with the use of the concept of transformation of the system in the parameterized form. The convergence of the identification error is guaranteed using the persistent excitation (PE) condition. Also, finite time convergence was assured using the terminal sliding mode. In [13], the author applied a sliding mode controller to stabilize uncertain time-delay chaotic systems. The proposed controller was robust against time-delays, parameter uncertainties and disturbances. The H-infinity theory has also been used to control time-delay systems. In [14], time-delays appeared in the network used in the feedback loop. The delay-dependent stability criterion was derived from the Lyapunov - Krasovskii function and the Linear Matrix Inequality (LMI). The $\mathrm{H}_{2}, \mathrm{H}-$ infinity and the LMI concepts have been used for discrete time delay uncertain systems. Authors in [15] used the past values of the states and the outputs, and were able to stabilize the system with time-varying delays. Finite time stability of time-delay systems has been investigated in [7, 16-18] with the utilization of the homogeneity theory. The observer design of time delay systems was used in [19] for a switched singular system where two design methods were used and in [20], a Luenberger-like observer has been used to estimate the unknown inputs for a large class of linear systems. The output regulation of timedelay systems has also been investigated in [21] by using the adaptive concept and the observer design using RBF neural network systems to approximate unknown functions. In [22] the well known Lyapunov-Krasovskii theorem was used to investigate the output stabilization for time-delay nonholonomic systems.

The simple MRAC of MIMO plants was first proposed in [23]. This class of algorithms does not require full state access or satisfaction of perfect model conditions. Asymptotic stability is ensured provided that the plant is Almost Strictly Positive Real (ASPR). Authors in [24] extended the original algorithm to a class of plants which violates this condition. This approach involved designing a supplementary feedforward filter to be included in parallel with the original plant resulting in a new augmented plant which had to satisfy the same strictly positive real condition. Unfortunately, the tracking error was not the true difference between the plant and the model outputs since it included the contribution of the supplementary feedforward filter which led to an asymptotically stable error [25-28]. The application of adaptive fuzzy control can be found in [33, 34]. The authors considered the internal model for controlling DCDC converters. Adaptive control is also used in many industrial fields, while authors in [35] used it for controlling UAV systems. 
Authors in [36] developed a saturated command for planar systems where the stabilization is achieved in finite time using just a simple proportional derivative corrector PD whose parameters are optimally adapted. This finite time stability is analyzed with Lyapunov's theory and homogeneity concept. Author in [37] aimed to replace a mechanical cam system with an electromagnetic actuator. The electromagnetic actuator creates a force which acts on the valve shaft and allows it to move linearly allowing the admission and exhaust of the explosion gas and therefore the combustion engine rotation. Electromagnetic force is generated by making a velocity measurement without a speed sensor. The position is deducted adaptively according to the estimated speed. This adaptive technique improves the efficiency of the mechanical engine and its longevity. The same author in [38] follows up this work and tries to remedy the problems encountered by the classic PD corrector in the presence of noise at high frequencies by optimally approximating the PD corrector parameters. After finding an actuator's model of electromagnetic valve actuator which replaces the classic mechanical valve actuator, the PD parameters are adjusted adaptively and on line with the variance minimization method.

\section{DIRECT MODEL REFERENCE ADAPTIVE CONTROL}

The model reference adaptive control is considered for the non-linear plant:

$$
\begin{aligned}
& \dot{x}_{p}(t)=A_{p} x_{p}(t)+A_{1} x_{p}(t-\tau(t))+B_{p} u_{p}(t)+f\left(x_{p}\right) \\
& y_{p}(t)=C_{p} x_{p}(t)
\end{aligned}
$$

where $x_{p}(t)$ is the $(n \times 1)$ state vector, $u_{p}(t)$ is the $(m \times 1)$ control vector, $\mathrm{y}_{p}(t)$ is the $(q \times 1)$ plant output vector, $f(t)$ is an $(n \times 1)$ vector of nonlinearities, $A_{p}, B_{p}$ are matrices with appropriate dimensions, and $\tau(t)$ is the time delay that verifies assumption (2) as stated below. We assume that the parameters of the linear part of the plant model are uncertain, i.e. only known within certain finite bounds. The range of the plant parameters is assumed to be known and bounded with:

$$
\begin{aligned}
& \underset{-i j}{a} \leq a_{p}(i, j) \leq \bar{a}_{i j}, i, j=1, \ldots, n \\
& b_{-i j} \leq b_{p}(i, j) \leq \bar{b}_{i j}, i, j=1, \ldots, n
\end{aligned}
$$

\section{- Assumption 1:}

The non-linear function $f(\mathrm{x})$ is Lipschitz in its arguments, that means $\left|f\left(x_{1}\right)-f\left(x_{2}\right)\right|<L\left|x_{1}-x_{2}\right|$ where $L>0$ is the constant of Lipschitz, $|()$.$| is the Euclidean norm and x_{1}, x_{2}$ belong to a compact set $\Omega \in R^{n}$.

\section{- Assumption 2}

The derivative of the delay system $\tau(t)$ verifies:

$$
\left|\frac{\mathrm{d} \tau(t)}{\mathrm{d} t}\right| \leq \tau_{1}
$$

The objective of this paper is to find, without explicit knowledge of $A_{p}, B_{p}$, and for non-linear $f\left(x_{p}\right)$, the control $u_{p}(t)$ such that the plant output vector $y_{p}(t)$ follows the reference model given by:

$$
\begin{aligned}
x_{m}(t) & =A_{m} x_{m}(t)+A_{m \tau} x_{m}(t-\tau)+B_{m} u_{m}(t) \\
& +B_{m \tau} u_{m}(t-\tau) \\
y_{m}(t) & =C_{m} x_{m}(t)
\end{aligned}
$$

The output $y_{m}$ is the desired response to the set point command $u_{m}$. The model incorporates the desired behavior of the plant, but its choice is not restricted. In particular, the order of the plant may be much larger than the order of the reference model. The ideal control law that generates perfect output tracking and ideal state trajectories is assumed to be a linear combination of the model states and the model input (see [29]). In our case, we suppose that the ideal state, its delay and the ideal input are related to the model state. Its delay and the model input are related by:

$$
\left[\begin{array}{l}
x_{p}^{*}(t) \\
u_{p}^{*}(t)
\end{array}\right]=\left[\begin{array}{ll}
S_{11} & S_{12} \\
S_{21} & S_{22}
\end{array}\right]\left[\begin{array}{l}
x_{m}(t) \\
u_{m}(t)
\end{array}\right]
$$

The perfect output tracking means that the ideal output $y^{*}(t)$ is equal to the output model $y_{m}(t)$ which means:

$$
\begin{aligned}
& y_{p}^{*}(t)=C_{p} x_{p}^{*}(t)=C_{p} S_{11} x_{m}(t)+C_{p} S_{12} u_{m}(t) \\
& =y_{m}(t)=C_{m} x_{m}(t)
\end{aligned}
$$

Taking into account (5.0) and that the ideal state $x^{*}(t)$ verifies this relation and the assumption that the command $u_{m}(t)$ is constant (in the case where the input is not a constant, we can always find a dynamic system to generate $u_{m}(t)$ with a constant input), the derivative of $x^{*}(t)$ can be written as:

$$
\begin{aligned}
& \frac{\mathrm{d} x_{p}^{*}(t)}{\mathrm{d} t}=\frac{\mathrm{d}}{\mathrm{d} t}\left(S_{11} x_{m}(t)+S_{12} u_{m}(t)\right) \\
= & S_{11}\left(A_{m} x_{m}(t)+A_{m \tau} x_{m}(t-\tau)+B_{m} u_{m}(t)+B_{m \tau} u_{m}(t-\tau)\right) \\
= & S_{11} A_{m} x_{m}(t)+S_{11} A_{m \tau} x_{m}(t-\tau)+S_{11} B_{m} u_{m}(t)+S_{11} B_{m \tau} u_{m}(t-\tau) \\
= & A_{p} x_{p}^{*}(t)+A_{1} x_{p}^{*}(t-\tau)+B_{p} u_{p}^{*}(t) \\
= & A_{p}\left(S_{11} x_{m}(t)+S_{12} u_{m}(t)\right)+A_{l}\left(S_{11} x_{m}(t-\tau)+\right. \\
& \left.S_{12} u_{m}(t-\tau)\right)+B_{p}\left(S_{21} x_{m}(t)+S_{22} u_{m}(t)\right)
\end{aligned}
$$

Using (5.1)-(5.2), we obtain the following algebraic system:

$$
\left\{\begin{array}{l}
S_{11} A_{m}=A_{p} S_{11}+B_{p} S_{21} \\
S_{11} B_{m}=A_{p} S_{12}+B_{p} S_{22} \\
S_{11} A_{m \tau}=A_{1} S_{11} \\
S_{11} B_{m \tau}=A_{1} S_{12} \\
C_{p} S_{11}=C_{m} \\
C_{p} S_{12}=0
\end{array}\right.
$$

which can be written as: 


$$
\left[\begin{array}{cc}
S_{11} A_{m} & S_{11} B_{m} \\
S_{11} A_{m \tau} & S_{11} B_{m \tau} \\
C_{m} & 0
\end{array}\right]=\left[\begin{array}{ll}
A_{p} & B_{p} \\
A_{1} & 0 \\
C_{p} & 0
\end{array}\right]\left[\begin{array}{ll}
S_{11} & S_{12} \\
S_{21} & S_{22}
\end{array}\right]
$$

In the system (6) we have more unknowns than equations, so the solution almost always exists. When $A_{l}, A_{m \tau}$ and $B_{m \tau}$ are null, then the system and the model are without delay and we get the equations given in [29]. The adaptive control law based on the extended Command Generator Tracker (CGT) approach is given by:

$$
u_{p}(t)=K_{e}(t) e_{y}(t)+K_{x}(t) x_{m}(t)+K_{u}(t) u_{m}(t)
$$

The adaptive law (7) has been applied for linear systems $[30,31]$. Here we aim to extend it to a linear time delay system described by (1) by adding a delay in the input and output in the model (4). The tracking error is given by: $e_{y}(t)=y_{m}(t)-y_{p}(t)$ and $K_{e}(t), K_{x}(t)$ and $K_{u}(t)$ are adaptive gains concatenated into matrix $K(t)$ as :

$$
K(t)=\left[K_{e}(t) K_{x}(t) K_{u}(t)\right]
$$

Defining the vector $r(t)\left(n_{r} \times 1\right)$ as:

$$
r(t)=\left[\begin{array}{lll}
\left(y_{m}(t)-y_{p}(t)\right)^{T} & x_{m}^{T}(t) & u_{m}^{T}(t)
\end{array}\right]^{T}
$$

the control $u_{p}(t)$ is written in a compact form as:

$$
u_{p}(t)=K(t) r(t)
$$

where

$$
\begin{gathered}
K(t)=K_{p}(t)+K_{I}(t) \\
K_{p}(t)=\left[y_{m}(t)-y_{p}(t)\right] \cdot r^{T}(t) T_{p}, T_{p} \geq 0 \\
\dot{K}_{I}(t)=\left[y_{m}(t)-y_{p}(t)\right] \cdot r^{T}(t) T_{i}, \quad T_{i}>0
\end{gathered}
$$

\section{STABILITY STUDY}

The first step of the demonstration is to design a positive definite quadratic form in the state variables $e_{x}(t)$ and $K_{I}(t)$ of the adaptive system. $T_{i}^{-1}$ is assumed to be a symmetric positive definite matrix. Then an appropriate choice of the LyapunovKrasovskii functional [32] is:

$$
\begin{aligned}
V= & e_{x}^{T} P e_{x}+\int_{t-\tau}^{t} e_{x}^{T}(\alpha) Q e_{x}(\alpha) d \alpha+ \\
& \operatorname{Tr}\left[S\left(K_{I}-\tilde{K}\right) T_{i}^{-1}\left(K_{I}-\tilde{K}\right)^{T} S^{T}\right]
\end{aligned}
$$

where $\operatorname{Tr}$ is the trace of a matrix. Its time derivative is:

$$
\begin{aligned}
\dot{V}= & \dot{e}_{x}^{T} P e_{x}+e_{x}^{T} P \dot{e}_{x}+e_{x}^{T}(t) Q e_{x}(t) \\
& -(1-\dot{\tau}) e_{x}^{T}(t-\tau) Q e_{x}(t-\tau)+ \\
& 2 \operatorname{Tr}\left[S\left(K_{I}-\tilde{K}\right) T_{i}^{-1} \dot{K}_{I} S^{T}\right]
\end{aligned}
$$

where $P, Q$ are symmetric positive definite matrices of size $n \times n, K$ is a $\mathrm{m} \times n$ matrix and $S$ is a non-singular $m \times m$ matrix.

Since the matrix $\tilde{K}$ appears only in the function $V$ and not in the control algorithm, it is called fictitious gain matrix. It has the same dimension as $K$ where:

$$
\tilde{K} \times r=\tilde{K}_{e} C_{p} e_{x}+\tilde{K}_{x} x_{m}+\tilde{K}_{u} u_{m}
$$

The four gains $\tilde{K}_{e}, \tilde{K}_{x}, \tilde{K}_{u}$ and $\tilde{K}_{x r}$ are as $\tilde{K}$ fictitious. Then we take the equation of the error using the fact that $e_{x}=x_{p}^{*}-x_{p}$ to find:

$$
\begin{aligned}
\dot{e}_{x}= & A_{p} x_{p}^{*}+A_{1} x_{p}^{*}(t-\tau)+B_{p} u_{p}^{*}+f\left(x_{p}^{*}\right)-A_{p} x_{p} \\
& -A_{1} x_{p}(t-\tau)-B_{p} u_{p}-f\left(x_{p}\right) \\
= & A_{p}\left[x_{p}^{*}-x_{p}\right]+A_{1}\left[x_{p}^{*}(t-\tau)-x_{p}(t-\tau)\right] \\
& +B_{p}\left[u_{p}^{*}-u_{p}\right]+f\left(x_{p}^{*}\right)-f\left(x_{p}\right) \\
= & A_{p} e_{x}+A_{1} e_{x}(t-\tau)+B_{p}\left[u_{p}^{*}-u_{p}\right]+f\left(x_{p}^{*}\right) \\
& -f\left(x_{p}\right)
\end{aligned}
$$

If we set: $d f=f\left(x_{p}^{*}\right)-f\left(x_{p}\right)$ and substitute $u_{p}^{*}$ from (5.0) and $u_{p}$ from (7), we get:

$$
\begin{aligned}
e_{x} & =A_{p} e_{x}+A_{1} e_{x}(t-\tau) \\
B_{p} & {\left[S_{21} x_{m}+S_{22} u_{m}-K_{x} x_{m}-K_{u} u_{m}-K_{e} C_{p} e_{x}\right]+d f } \\
= & A_{p} e_{x}+A_{1} e_{x}(t-\tau)+ \\
& B_{p}\left[S_{21} x_{m}+S_{22} u_{m}-K_{I} r-C_{p} e_{x} r^{T} T_{p} r\right]+d f
\end{aligned}
$$

Then the adaptive system is described by:

$$
\begin{gathered}
\dot{e}_{x}=A_{p} e_{x}+A_{1} e_{x}(t-\tau) \\
+B_{p}\left[S_{21} x_{m}+S_{22} u_{m}-K_{I} r-C_{p} e_{x} r^{T} T_{p} r\right]+d f \\
\dot{K}_{I}=C_{p} e_{x} r^{T} T_{i}
\end{gathered}
$$

Substituting (19) and (20) in (15), we get:

$$
\begin{aligned}
\dot{V} & =\left[\begin{array}{l}
A_{p} e_{x}+A_{1} e_{x}(t-\tau)+B_{p}\left(S_{21} x_{m}+S_{22} u_{m}-K_{I} r\right]^{T} P e_{x} \\
-C_{p} e_{x} r^{T} T_{p} r
\end{array}\right] \\
& +e_{x}^{T} P\left[\begin{array}{l}
A_{p} e_{x}+A_{1} e_{x}(t-\tau)+B_{p}\left(S_{21} x_{m}+S_{22} u_{m}\right. \\
-K_{I} r-C_{p} e_{x} r^{T} T_{p} r
\end{array}\right] \\
& +2 \operatorname{Tr}\left[S\left(K_{I}-\tilde{K}\right) T_{i}^{-1}\left(C_{p} e_{x} r^{T} T_{i}\right)^{T} S^{T}\right]+ \\
& e_{x}^{T}(t) Q e_{x}(t)-(1-\tau) e_{x}^{T}(t-\tau) Q e_{x}(t-\tau)+d f
\end{aligned}
$$

We can write it as: 


$$
\begin{aligned}
\dot{V}= & e_{x}^{T} A_{p}^{T} P e_{x}+e_{x}^{T}(t-\tau) A_{1}^{T} P e_{x}+ \\
& \left(x_{m}^{T} S_{21}^{T} B_{p}^{T}+u_{m}^{T} S_{22}^{T} B_{p}^{T}-r^{T} K_{I}^{T} B_{p}^{T}-r^{T} T_{p}^{T} r e_{x}^{T} C_{p}^{T} B_{p}^{T}\right) P e_{x}+ \\
& e_{x}^{T} P A_{p} e_{x}+e_{x}^{T} P A_{1} e_{x}(t-\tau)+ \\
& e_{x}^{T} P B_{p}\left(S_{21} x_{m}+S_{22} u_{m}-K_{I} r-C_{p} e_{x} r^{T} T_{p} r\right)+ \\
& 2 T r\left[S\left(K_{I}-\tilde{K}\right) T_{i}^{-1} T_{i}^{T} r e_{x}^{T} C_{p}^{T} S^{T}\right]+ \\
& e_{x}^{T}(t) Q e_{x}(t)-(1-\dot{\tau}) e_{x}^{T}(t-\tau) Q e_{x}(t-\tau)+d f
\end{aligned}
$$

Knowing that for two vectors $U(l, 1)$ and $V(1, l)$ have $\operatorname{Tr}[U . V]=V . U$ therefore:

$$
\begin{aligned}
\dot{V} & =e_{x}^{T}\left(P A_{p}+A_{p}^{T} P+Q\right) e_{x}+e_{x}^{T}(t-\tau) A_{1}^{T} P e_{x}+ \\
& e_{x}^{T} P A_{1} e_{x}(t-\tau)+e_{x}^{T} P B_{p} S_{21} x_{m}+e_{x}^{T} P B_{p} S_{22} u_{m} \\
& -e_{x}^{T} P B_{p} K_{I} r-e_{x}^{T} P B_{p} C_{p} e_{x} r^{T} T_{p} r+x_{m}^{T} S_{21}^{T} B_{p}^{T} P e_{x} \\
& +u_{m}^{T} S_{22}^{T} B_{p}^{T} P e_{x}-r^{T} K_{I}^{T} B_{p}^{T} P e_{x}-r^{T} T_{p}^{T} r e_{x}^{T} C_{p}^{T} B_{p}^{T} P e_{x} \\
& +2 e_{x}^{T} C_{p}^{T} S^{T} S\left(K_{I}-\tilde{K}\right) r+d f \\
& -(1-\tau) e_{x}^{T}(t-\tau) Q e_{x}(t-\tau)
\end{aligned}
$$

which means that:

$$
\begin{aligned}
\dot{V} & =e_{x}^{T}\left(P A_{p}+A_{p}^{T} P+Q\right) e_{x}+e_{x}^{T}(t-\tau) A_{1}^{T} P e_{x} \\
& +e_{x}^{T} P A_{1} e_{x}(t-\tau)+2 e_{x}^{T} P B_{p}\left(S_{21} x_{m}+S_{22} u_{m}\right) \\
& -2 e_{x}^{T} P B_{p} C_{p} e_{x} r^{T} T_{p} r+2 e_{x}^{T}\left[C_{p}^{T} S^{T} S-P B_{p}\right] K_{I} r \\
& -2 e_{x}^{T} C_{p}^{T} S^{T} S \tilde{K} r-(1-\tau) e_{x}^{T}(t-\tau) Q e_{x}(t-\tau)+d f
\end{aligned}
$$

By setting: $C_{p}=G B_{p}^{T} P$ and $G=\left(S^{T} S\right)^{-1}$, the derivative of the Lyapunov function becomes:

$$
\begin{aligned}
& \dot{V}=e_{x}^{T}\left(P A_{p}+A_{p}^{T} P+Q\right) e_{x}+e_{x}^{T}(t-\tau) A_{1}^{T} P e_{x} \\
& +e_{x}^{T} P A_{1} e_{x}(t-\tau)+2 e_{x}^{T} P B_{p}\left(S_{21} x_{m}+S_{22} u_{m}\right) \\
& -2 e_{x}^{T} P B_{p}\left(S^{T} S\right)^{-1} B_{p}^{T} P e_{x} r^{T} T_{p} r-2 e_{x}^{T} C_{p}^{T} S^{T} S \tilde{K} r \\
& -(1-\tau) e_{x}^{T}(t-\tau) Q e_{x}(t-\tau)+d f
\end{aligned}
$$

Substituting $\tilde{K}_{r}=\tilde{K}_{e} C_{p} e_{x}+\tilde{K}_{x} x_{m}+\tilde{K}_{u} u_{m}$ in (24) we get:

$$
\begin{aligned}
\dot{V} & = \\
e_{x}^{T} & {\left[P\left(A_{p}-B_{p} \tilde{K}_{e} C_{p}\right)+\left(A_{p}-B_{p} \tilde{K}_{e} C_{p}\right)^{T} P+Q\right] e_{x} } \\
& +e_{x}^{T}(t-\tau) A_{1}^{T} P e_{x}+e_{x}^{T} P A_{1} e_{x}(t-\tau) \\
& -2 e_{x}^{T} P B_{p}\left(S^{T} S\right)^{-1} B_{p}^{T} P e_{x} r^{T} T_{p} r \\
& +2 e_{x}^{T} P B_{p}\left[\left(S_{21}-\tilde{K}_{x}\right) x_{m}+\left(S_{22}-\tilde{K}_{u}\right) u_{m}\right] \\
& -(1-\tau) e_{x}^{T}(t-\tau) Q e_{x}(t-\tau)+d f
\end{aligned}
$$

Thus, if we set:

$$
\left[\left(S_{21}-\tilde{K}_{x}\right) x_{m}+\left(S_{22}-\tilde{K}_{u}\right) u_{m}\right]=0
$$

or $\tilde{K}_{x}=S_{21}$ and $\tilde{K}_{u}=S_{22}$ (none of which is required for implementation), the derivative of $V$ becomes:

$$
\begin{aligned}
& \dot{V}=e_{x}^{T}\left[P\left(A_{p}-B_{p} \tilde{K}_{e} C_{p}\right)+\left(A_{p}-B_{p} \tilde{K}_{e} C_{p}\right)^{T} P+Q\right] e_{x} \\
& \quad e_{x}^{T}(t-\tau) A_{1}^{T} P e_{x}+e_{x}^{T} P A_{1} e_{x}(t-\tau) \\
& -2 e_{x}^{T} P B_{p}\left(S^{T} S\right)^{-1} B_{p}^{T} P e_{x} r^{T} T_{p} r \\
& -(1-\tau) e_{x}^{T}(t-\tau) Q e_{x}(t-\tau)+d f
\end{aligned}
$$

Taking into account the Assumption 2, the derivative of the Lyapunov function verifies (27):

$$
\begin{aligned}
& \dot{V} \leq\left[\begin{array}{l}
e_{x}(t) \\
e_{x}(t-\tau)
\end{array}\right]^{T}\left[\begin{array}{cc}
Q_{1} & P A_{1} \\
A_{1}^{T} P & -\left(1-\tau_{1}\right) Q
\end{array}\right]\left[\begin{array}{l}
e_{x}(t) \\
e_{x}(t-\tau)
\end{array}\right] \\
& -2 e_{x}^{T} P B_{p}\left(S^{T} S\right)^{-1} B_{p}^{T} P e_{x} r^{T} T_{p} r+d f
\end{aligned}
$$

with

$$
Q_{1}=P\left(A_{p}-B_{p} \tilde{K}_{e} C_{p}\right)+\left(A_{p}-B_{p} \tilde{K}_{e} C_{p}\right)^{T} P+Q
$$

From (12), $T_{p}$ is positive semi-definite, so (27) becomes:

$$
\dot{V} \leq\left[\begin{array}{l}
e_{x}(t) \\
e_{x}(t-\tau)
\end{array}\right]^{T}\left[\begin{array}{cc}
Q_{1} & P A_{1} \\
A_{1}^{T} P & -\left(1-\tau_{1}\right) Q
\end{array}\right]\left[\begin{array}{l}
e_{x}(t) \\
e_{x}(t-\tau)
\end{array}\right]+d f
$$

Let's take:

$$
E_{x}=\left[\begin{array}{l}
e_{x}(t) \\
e_{x}(t-\tau)
\end{array}\right] \text { and } Q_{2}=-\left[\begin{array}{cc}
Q_{1} & P A_{1} \\
A_{1}^{T} P & -\left(1-\tau_{1}\right) Q
\end{array}\right]
$$

Then (29) is rewritten as:

$$
\dot{V} \leq-E_{x}^{T} Q_{2} E_{x}+d f
$$

When $d f$ is equal to zero, the error is asymptotically stable if and only if $Q_{2}=Q_{2}^{T}$ is positive semi-definite. In the case where $d f$ is different from zero and satisfies the Assumption 1, then the derivative of the Lyapunov function verifies:

$$
\begin{aligned}
\dot{V} & \leq-E_{x}^{T} Q_{2} E_{x}+|d f| \leq-E_{x}^{T} Q_{2} E_{x}+L\left|x-x^{*}\right|=-E_{x}^{T} Q_{2} E_{x}+L\left|e_{x}\right| \\
& \leq-\lambda_{\min }\left(Q_{2}\right)\left|E_{x}\right|^{2}+L\left|e_{x}\right| \leq-\lambda_{\min }\left(Q_{2}\right)\left|e_{x}\right|^{2}+L\left|e_{x}\right| \leq 0 \\
& \Rightarrow\left|e_{x}\right| \geq \frac{L}{\lambda_{\text {min }}\left(Q_{2}\right)}
\end{aligned}
$$

where $\lambda_{\text {min }}\left(Q_{2}\right)$ stands for the lowest eigenvalue of $Q_{2}$ which is a positive number since $Q_{2}=Q_{2}^{T} \geq 0$. The last inequality implies that the error $e_{x}$ is ultimately uniformly stable, which means that it belongs to a compact set around the origin. This set can be rendered much lower if we select $\lambda_{\text {min }}\left(Q_{2}\right)$ to be 
large. One choice is given by $Q_{2}=\alpha . I$ where $I$ is the identity matrix and $\alpha$ is a scalar positive number. Finally the derivative of the Lyapunov function is negative definite in $e_{x}$ if $\left|e_{x}\right| \geq L / \lambda_{\min }\left(Q_{2}\right)$. Since $V(t)$ is a positive definite function, then the vector $e_{x}(t)$ and the matrix $K_{I}(t)$ are bounded. We summarize the stability concept in the following theorem:

- Theorem:

The control given by (10), and the adaptive laws given by (11), (12) and (13) applied to the non-linear uncertain system (1) that verifies the Assumption 1 lead to a asymptotically stable error between the system and the model if and only if there are two $P, Q$ matrices $P=P^{T}>0$ and $Q=Q^{T} \geq 0$ such that:

1) The matrix

$$
Q_{2}=-\left[\begin{array}{lc}
H & P A_{1} \\
A_{1}^{T} P & -\left(1-\tau_{1}\right) Q
\end{array}\right]
$$

where

$$
H=P\left(A_{p}-B_{p} \tilde{K}_{e} C_{p}\right)+\left(A_{p}-B_{p} \tilde{K}_{e} C_{p}\right)^{T} P+Q
$$

is positive semi-definite for some matrix $\tilde{K}_{e}$.

2) $P B_{p}=(G C)^{T}, G=\left(S^{T} S\right)^{-1}$, for a non-singular matrix $S$.

3) $\left|f\left(x_{1}\right)-f\left(x_{2}\right)\right|<L\left|x_{1}-x_{2}\right|, \quad L>0 \quad x_{1}, x_{2} \in R^{2}$

$$
\begin{aligned}
& {\left[P\left(A_{p}-B_{p} \tilde{K}_{e} C_{p}\right)+\left(A_{p}-B_{p} \tilde{K}_{e} C_{p}\right)^{T} P\right]=-Q} \\
& =-\alpha I \\
& P B_{p}=(G C)^{T} \quad T_{p} \geq 0, \quad T_{i}>0, G=G^{T}>0 \\
& \left|f\left(x_{1}\right)-f\left(x_{2}\right)\right|<L\left|x_{1}-x_{2}\right|, \quad L>0 \quad x_{1}, x_{2} \in R^{2},
\end{aligned}
$$

where $\alpha \in R^{+}$, and $I$ is the identity matrix.

These relations imply that the feedback system is SPR for large $\alpha$, and so that the original linear system is ASPR.

\section{Simulation}

During simulations, it is required that the output of the system tracks the output reference. The controlled system is given by:

$$
\begin{aligned}
& \dot{x}_{p}(t)=A_{p} x_{p}(t)+A_{1} x_{p}(t-\tau(t))+B_{p} u_{p}(t) \\
& y_{p}(t)=C_{p} x_{p}(t)
\end{aligned}
$$

with $A_{p}=\left[\begin{array}{ll}1 & 3 \\ 3 & 4\end{array}\right], A_{1}=\left[\begin{array}{ll}5 & 6 \\ 6 & 8\end{array}\right], B=\left[\begin{array}{l}3 \\ 4\end{array}\right], C=\left[\begin{array}{ll}5 & 6\end{array}\right]$.

The transfer function of the reference model is given by:

$$
G_{m}(s)=\frac{2}{s+1}
$$

The eigenvalues of $A_{p}$ and $A_{1}$ are given by: $\lambda\left(A_{p}\right)=\{-0.85 ; 5.85\}$ and $\lambda\left(A_{1}\right)=\{0.31 ; 12.68\}$ which means that the two matrices are instable. The model input is illustrated in Figure 1, where $u_{m}=1$ from 0 to $20 \mathrm{~s}$ and $u_{m}=-1$ from $20 \mathrm{~s}$ to $40 \mathrm{~s}$. From $40 \mathrm{~s}$ to $60 \mathrm{~s}$ we have chosen a sinusoidal input given by $u_{m}(t)=2 \sin (t)$ and from $60 \mathrm{~s}$ to $100 \mathrm{~s}$, another sinusoidal input, $u_{m}(t)=\sin (\mathrm{t} / 3)$, was selected.

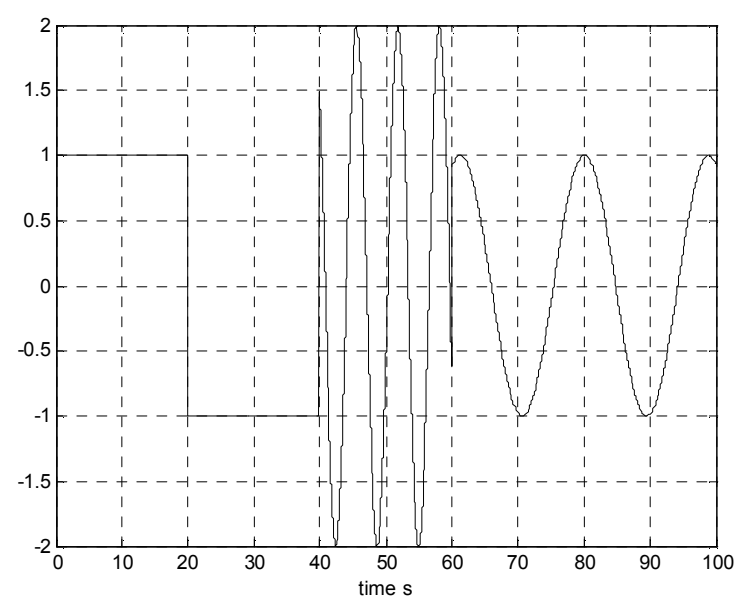

Fig. 1. The model input $u_{m}(t)$

\section{A. Case 1: Without Perturbation}

In this case we supposed that the controlled system is not affected by noise measurement and actuator failure. The adjustable parameter is chosen to be $T_{p}=T_{i}=1 \times I_{3,3}$ where $I_{3,3}$ is the identity matrix of order 3 . Figure 2 shows the two outputs where the output of the controlled system tracks the reference whatever is the model input. The controlled system is given in Figure 3 where we can see that this command has the same form as the model input. The four gains are presented in Figure 4. Note that these gains are used to construct the controlled system input (see (11), (12) and (13)). These gains are bounded so the controlled system input is also bounded.

\section{B. Case 2: With Perturbation and $T_{p}=T_{i}=1 \times I_{3,3}$}

In this case, a noise measurement and an actuator fault are added, so the perturbed system is given by:

$$
\begin{aligned}
& \dot{x}_{p}(t)=A_{p} x_{p}(t)+A_{1} x_{p}(t-\tau(t))+B_{p}\left(u_{p}(t)+d(t)\right) \\
& y_{p}(t)=C_{p} x_{p}(t)+n(t)
\end{aligned}
$$

where $d(t)$ is the actuator fault given by $d(t)=1 \sin (2 t)$, and $n(t)$ represents the measurement noise given by $n(t)=1 \sin (4 t)$. The adjustable parameters are taken as in the previous case. Figure 5 illustrates the outputs of the system and the model. It is clear that the tracking has been deteriorated due to the perturbation that has affected the system. Figure 6 represents the control input where the controller is doing a big effort in order to damp the effect of the perturbation and let the system output track the reference model. 


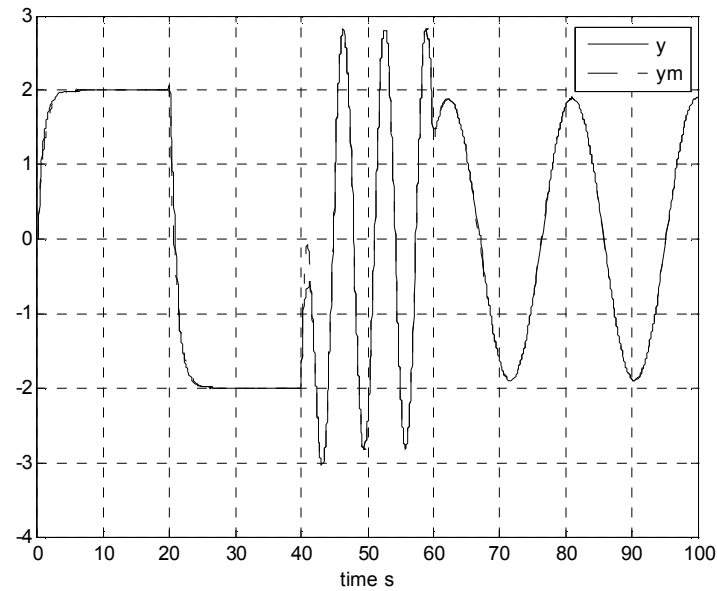

Fig. 2. Outputs of the system and the model without perturbation

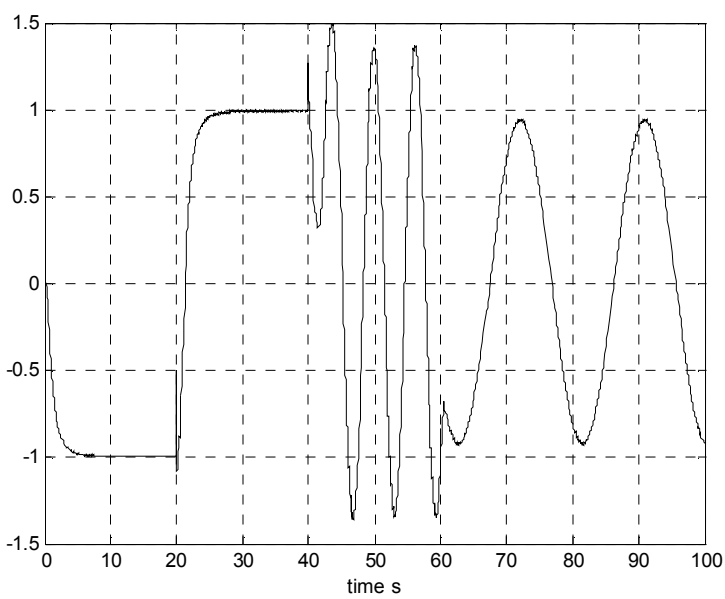

Fig. 3. The system command $u(t)$

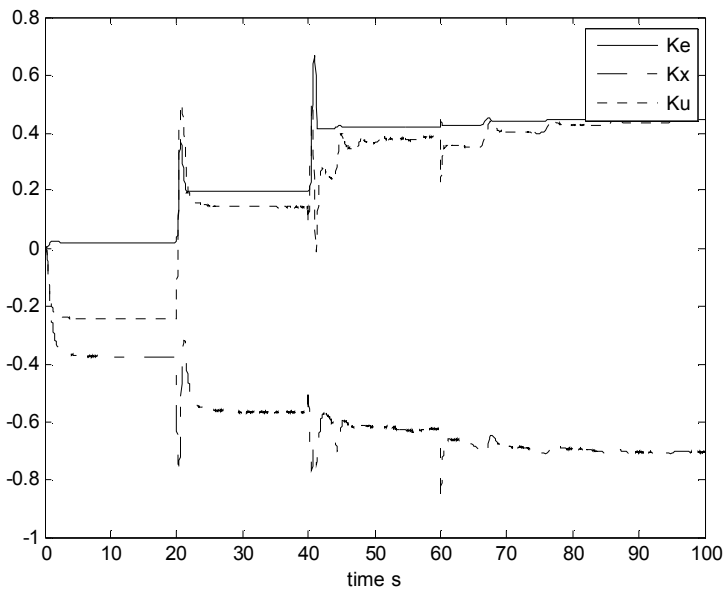

Fig. 4. The gains $K_{e}, K_{x}$, and $K_{u}$

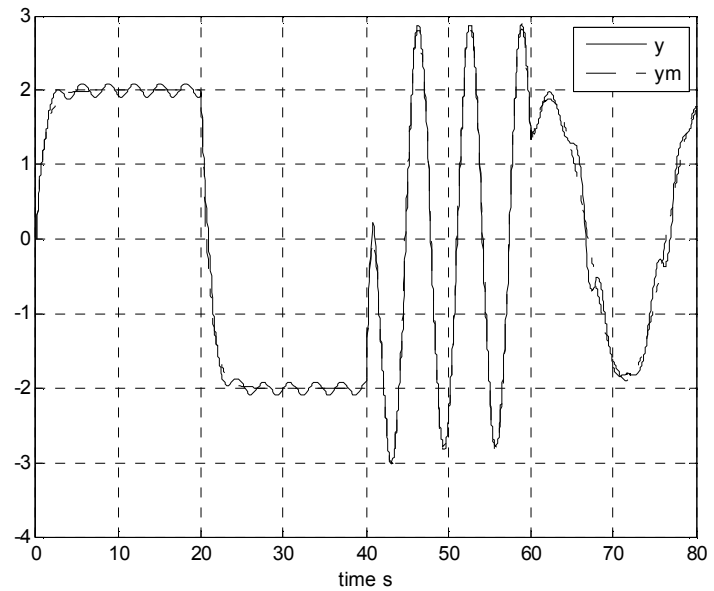

Fig. 5. Outputs of the system and the model with perturbation $T_{p}=T_{i}=1 \times I_{3,3}$

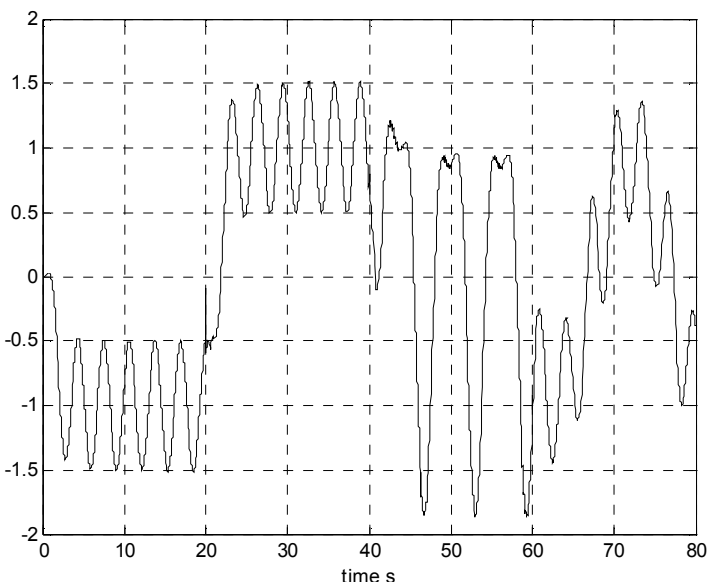

Fig. 6. The system command $u(t)$

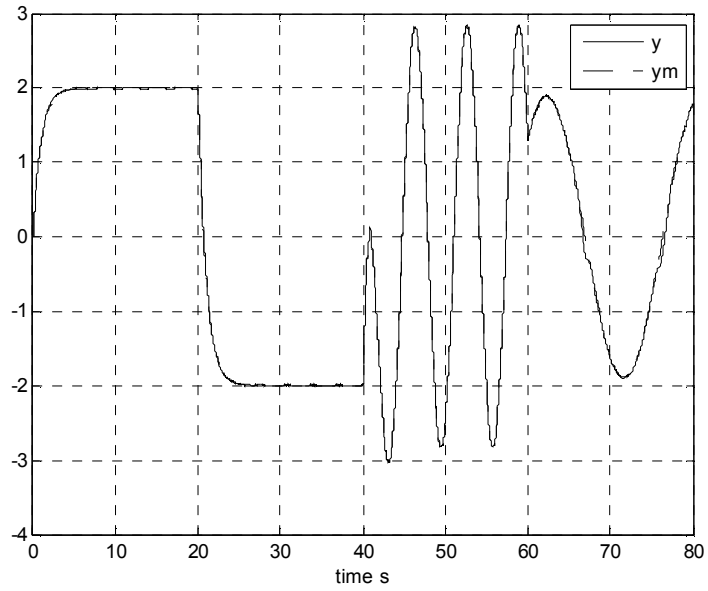

Fig. 7. Outputs of the system and the model with perturbation $T_{p}=T_{i}=10 \times I_{3,3}$ 
C. Case 3: With Perturbation and $T_{p}=T_{i}=10 \times I_{3,3}$

In this case and in order to overcome the drawback that has appeared in the previous case, we augmented the adjustable parameter $T_{p}, T_{i}$ as $T_{p}=T_{i}=10 \times I_{3,3}$. Figure 7 shows a perfect tracking compared to Figure 5 and Figure 8 shows the effect of the controller in overcoming the perturbation and letting the system output track the reference model. Note that this command is bounded and does not represent a high oscillation. Figure 9 presents the gains that are bounded and they are adjusted to construct the system input.

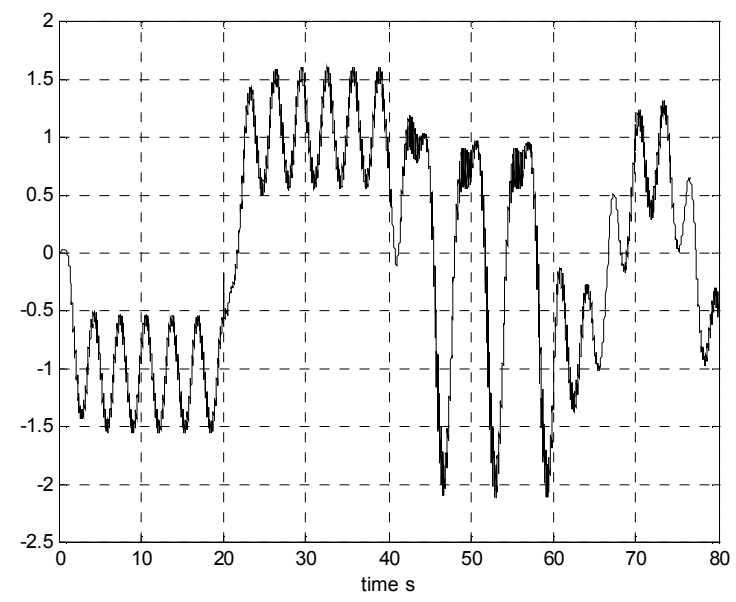

Fig. 8. The system command $u(t)$

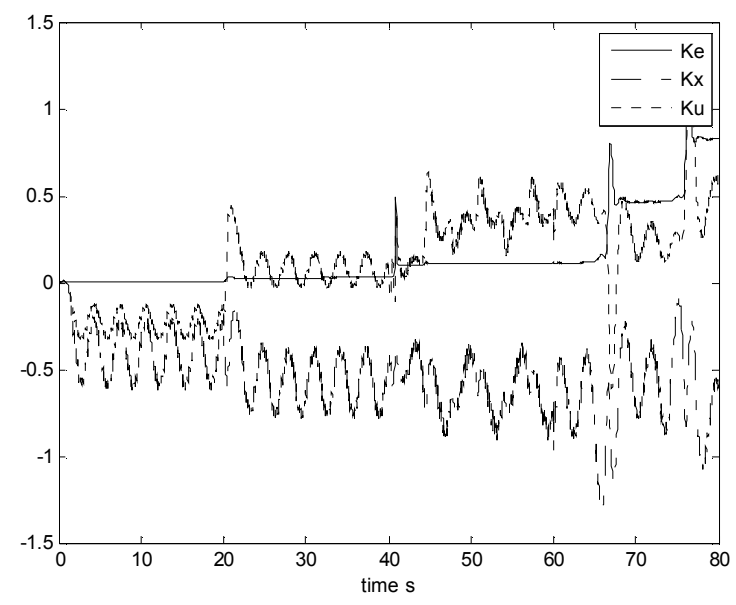

Fig. 9. The gains $K_{e}, K_{x}$, and $K_{u}$

\section{CONCLUSION}

This paper presents an adaptive command applied for a perturbed time delay system. The Lyapunov's theory has been addressed in order to achieve a robust command against the uncertainty which is inherent in all real systems. The simulation results confirm the robustness of the developed command.

\section{REFERENCES}

[1] E. Fridman, "Tutorial on Lyapunov-based methods for time-delay systems", European Journal of Control, Vol. 20, No. 6, pp. 271-283, 2014

[2] K. Gu, V. L. Kharitonov, J. Chen, Stability of time-delay systems, Springer, 2003

[3] M. Wu, Y. He, J. H. She, Stability analysis and robust control of timedelay systems, Springer, 2010

[4] X. P. Chen, H. Dai, "Stability analysis of time-delay systems using a contour integral method", Applied Mathematics and Computation, Vol. 273, pp. 390-397, 2016

[5] M. Hashemi, J. Ghaisari, J. Askari, "Adaptive control for a class of MIMO nonlinear time delay systems against time varying actuator failures", ISA Transactions, Vol. 57, pp. 23-42, 2015

[6] Z. Zhang, C. Lin, B. Chen, "New stability and stabilization conditions for T-S fuzzy systems with time delay", Fuzzy Sets Systems, Vol. 263, pp. 82-91, 2015

[7] J. Song, S. He, "Finite-time robust passive control for a class of uncertain Lipschitz nonlinear systems with time-delays", Neurocomputing, Vol. 159, pp. 275-281, 2015

[8] S. H. Tsai, Y. A. Chen, J. C. Lo, "A novel stabilization condition for a class of T-S fuzzy time-delay systems", Neurocomputing, Vol. 175, pp. 223-232, 2015

[9] Y. D. Song, H. Zhou, X. Su, L. Wang, "Pre-specified performance based model reduction for time-varying delays systems in fuzzy frame work", Information Sciences, Vol. 328, pp. 206-221, 2016

[10] D. J. Wang, "A PID controller set of guaranteeing stability and gain and phase margins for time-delay systems", Journal of Process Control, Vol. 22, No. 7, pp. 1298-1306, 2012

[11] D. B. Pietri, J. Chauvin, N. Petit, "Adaptive control scheme for uncertain time-delay systems”, Automatica, Vol. 48, No. 8, pp. 1536-1552, 2012

[12] J. Na, X. Ren, Y. Xia, "Adaptive parameter identification of linear SISO systems with unknown time-delay”, Systems \& Control Letters, Vol. 66, pp. 43-50, 2014

[13] M. C. Pai, "Chaotic sliding mode controllers for uncertain time-delay chaotic systems with input nonlinearity", Applied Mathematics and Computation, Vol. 271, pp. 757-767, 2015

[14] Y. Zhang, Q. Wang, C. Dong, Y. Jiang, "Hळ output tracking control for flight control systems with time-varying delay", Chinese Journal of Aeronautics, Vol. 26, No. 5, pp. 1251-1258, 2013

[15] L. Frezzatto, M. J. Lacerda, R. C. L. F. Oliveira, P. L. D. Peres, "Robust $\mathrm{H} 2$ and $\mathrm{H} \infty$ memory filter design for linear uncertain discrete-time delay systems", Signal Processing, Vol. 117, pp. 322-332, 2015

[16] V. Andrieu, L. Praly, L. Astolfi, "Homogeneous approximation, recursive observer design, and output feedback", SIAM Journal on Control and Optimization, Vol. 47, No. 4, pp. 1814-1850, 2008

[17] A. Polyakov, "Nonlinear feedback design for fixed-time stabilization of linear control systems", IEEE Transactions on Automatic Control, Vol. 57, No. 8, pp. 2106-2110, 2012

[18] D. Efimov, A. Polyakov, E. Fridman, W. Perruquetti, J. P. Richard, "Comments on finite-time stability of time-delay systems", Automatica, Vol. 50, No. 7, pp. 1944-1947, 2014

[19] J. Lin, Z. Gao, "Observers design for switched discrete-time singular time-delay systems with unknown inputs", Nonlinear Analysis: Hybrid Systems, Vol. 18, pp. 85-99, 2015

[20] G. Zheng, F. J. Bejarano, W. Perruquetti, J. P. Richard, "Unknown input observer for linear time-delay systems", Automatica, Vol. 61, pp. 35-43, 2015

[21] C. Hua, G. Liu, L. Zhang, X. Guan, "Output feedback tracking control for nonlinear time-delay systems with tracking errors and input constraints", Neurocomputing, Vol. 173, pp. 751-758, 2016

[22] Y. Q. Wu, Z. G. Liu, “Output feedback stabilization for time-delay nonholonomic systems with polynomial conditions", ISA Transactions, Vol. 58, pp. 1-10, 2015 
[23] K. Sobel, H. Kaufman, L. Mabius, "Implicit adaptive control for a class of MIMO systems", IEEE Transactions on Aerospace and Electronic Systems, Vol. 18, No. 5, pp. 576-590, 1982

[24] S. Ozcelik, H. Kaufman, "Robust direct model reference adaptive controllers", 34th IEEE Conference on Decision and Control, New Orleans, USA, December 13-15, 1995

[25] G. W. Neat, H. Kaufman, R. Steinvorth, "Comparison and extension of a direct model reference adaptive control procedure", International Journal of Control, Vol. 55, No. 4, pp. 945-967, 1992

[26] I. Bar-kana, "Positive-realness in multivariable stationary linear systems", Journal of the Franklin Institute, Vol. 328, No. 4, pp. 403-417, 1991

[27] I. Barkana, M. C. M. Teixeira, L. Hsu, "Mitigation of symmetry condition in positive realness for adaptive control", Automatica, Vol. 42, No. 9, pp. 1611-1616, 2006

[28] I. Barkana, "Gain conditions and convergence of simple adaptive control", International Journal of Adaptive Control and Signal Processing, Vol. 19, No. 1, pp. 13-40, 2005

[29] J. Broussard, M. O'Brien, "Feedforward control to track the output of a forced model", IEEE Transactions on Automatic Control, Vol. 25, No. 4, pp. 851-853, 1980

[30] D. A. Torrey, Y. Sozer, H. Kaufman, "Direct model reference adaptive control of permanent magnet brushless DC motors", IEEE International Conference on Control Applications, Hartford, USA, October 5-7, 1997

[31] H. Kaufman, G. W. Neat, "Asymptotically stable multiple-input multiple-output direct model reference adaptive controller for processes not necessarily satisfying a positive real constraint", International Journal of Control, Vol. 58, No. 5, pp. 1011-1031, 1993

[32] M. Darouach, "Linear functional observers for systems with delays in state variables", IEEE Transactions on Automatic Control, Vol. 46, No. 3, pp. 491-496, 2001

[33] K. Behih, K. Benmahammed, Z. Bouchama, M. N. Harmas, "Real-time investigation of an adaptive fuzzy synergetic controller for a DC-DC buck converter", Engineering, Technology \& Applied Science Research, Vol. 9, No. 6, pp. 4984-4989, 2019

[34] Z. R. Labidi, H. Schulte, A. Mami, "A model-based approach of DC-DC converters dedicated to controller design applications for photovoltaic generators", Engineering, Technology \& Applied Science Research, Vol. 9, No. 4, pp. 4371-4376, 2019

[35] K. Mokhtari, A. Elhadri, M. Abdelaziz, "A passivity-based simple adaptive synergetic control for a class of nonlinear systems", International Journal of Adaptive Control and Signal Processing, Vol. 33, No. 9, pp. 1359-1373, 2019

[36] Y. Su, C. Zheng, P. Mercorelli, "Global finite-time stabilization of planar linear systems with actuator saturation", IEEE Transactions on Circuits and Systems II: Express Briefs, Vol. 64, No. 8, pp. 947-951, 2017

[37] P. Mercorelli, "An adaptive and optimized switching observer for sensorless control of an electromagnetic valve actuator in camless internal combustion engines", Asian Journal of Control, Vol. 16, No. 4, pp. 959-973, 2014

[38] P. Mercorelli, "Robust adaptive soft landing control of an electromagnetic valve actuator for camless engines", Asian Journal of Control, Vol. 18, No. 4, pp. 1299-1312, 2016 